\title{
Feature extraction for brain tumour analysis and classification: a review
}

\section{Rajat Mehrotra*}

Department of Electrical \& Electronics Engineering, GL Bajaj Institute of Technology \& Management, Greater Noida, India

Email: rajatlmp@gmail.com

*Corresponding author

\section{M.A. Ansari}

Department of Electrical Engineering,

School of Engineering,

Gautam Buddha University,

Greater Noida, India

Email: ma.ansari@gbu.ac.in

\begin{abstract}
The most vital organ of the human body is brain which organises the whole of the sensory system. The presented paper provides a knowledgeable perception of diverse strategies utilised by various researchers for segmentation and identification of brain tumour (BT) using distinct methods and approaches. Among various imaging modalities, MRI images are acknowledged here as an input of superior quality for the purpose of conducting research when compared to other existing modern practices for superior and more precise outcome. Biomedical image processing has witnessed exponential growth, and has been a multidisciplinary area of research attracting experts not only from medical field but from various other fields of engineering and sciences. Computer-aided diagnostic processing has already become an important part of clinical routine.
\end{abstract}

Keywords: magnetic resonance imaging; MRI; classification; segmentation; feature extraction; fuzzy C-means; FCMs; neuro fuzzy; genetic algorithm; GA; grey-level co-occurrence matrix; GLCM; histogram of oriented gradient; HoG; linear binary pattern; LBP; discrete wavelet transform; DWT.

Reference to this paper should be made as follows: Mehrotra, R. and Ansari, M.A. (2020) 'Feature extraction for brain tumour analysis and classification: a review', Int. J. Digital Signals and Smart Systems, Vol. 4, Nos. 1/2/3, pp.199-218.

Biographical notes: Rajat Mehrotra is an Assistant Professor in the Electrical \& Electronics Engineering Department at GL Bajaj Institute of Technology \& Management, Greater Noida, India. He received his BTech in Electrical and Electronics Engineering from the Dr. A.P.J. Abdul Kalam Technical University, Lucknow (formerly UPTU), in 2008 and MTech in Telecommunication Engineering from the same university, in 2014. His research interests are in digital image processing, biomedical imaging and deep learning. Currently, he is pursuing his research work in the School of Engineering at Gautam Buddha University, Greater Noida. 
M.A. Ansari received his BTech in Electrical Engineering from the Aligarh Muslim University, India, in 1998, and MTech in Electrical Engineering and $\mathrm{PhD}$ in Digital Image Processing from Indian Institute of Technology, Roorkee, India, in 2001 and 2009, respectively. He has taught in several national and international universities globally. $\mathrm{He}$ is currently associated with the Department of Electrical Engineering, School of Engineering, Gautam Buddha University, Greater Noida, NCR Delhi, India. His research interests include digital image processing, medical imaging, biomedical instrumentation and control and bio-energy. He is a senior member of the IEEE and has published more than 100 papers in national and international journals and conferences and has guided several MTech dissertations and PhDs.

\section{Introduction}

Brain, the cardinal agency of the human anatomy and controls the overall human nervous system. It controls functions of the heart, breathing, walking, discerning ability, alertness and asleep, etc. Hence, brain has an important role to play in the nervous system of humans. Biomedical image processing has witnessed exponential growth and has been a multidisciplinary area of research attracting experts not only from the medical field but from applied mathematics, computer sciences, engineering, statistics, physics, etc. Computer-aided diagnostic processing has already become an important part of clinical routine. Followed by a flash of fresh development of high-end technologies and making usage of varied imaging modalities, fresh tasks arise; for instance, how a significant volume of images can be analysed and processed so as to produce high-quality information for treatment and diagnoses of disease.

Benign and harmful are two sorts of brain tumours (BTs), a standout amongst which will be a malignant one which keeps on growing. Harmful BT might be further separated under two classes as primary tumour and secondary tumour. Elementary BT develops in the brain and auxiliary kind BT Initially develops in the brain and then spreads to the cerebrum and begins influencing them. World Health Organization had recognised approximately 120 sorts of tumours. According to American Cancer Society (2016), the dangerous tumour that develops in the cerebrum and spinal cord had influenced 23,800 adults and 100,000 kids in the USA in a year. An effective way of treating such BT's is the surgical treatment and medical imaging modalities like computed tomography (CT) scan, magnetic resonance imaging (MRI) scans, positron emission tomography (PET) scans, etc. are used to detect such BT's. The vast majority of MRI test utilised for making a diagnosis of a brain with radio wave pulses of energy. The medicinal experts diagnosed and conclude whether the organ is normal or not. Detecting BT may be precise, requesting more testing assignment to detect them in the early phases because of their diverse appearances, dissimilar sizes, metabolism, shapes, noisy images, diffused and overlap because of the tentacle-like structure as per Meera and Sindhu (2015) and Poonam (2014). The division may be those principle preprocessing ventures for the identification of BTs. It enhances the performance and exactness of automatically detecting the BT. Various kinds of machine-based learning methods and procedures are considered to MRI and CT scan to identify the BT during the first phase.

The programmed BT framework might comprise of stages demonstrated in Figure 2. The image acquisition utilises MRI scans, CT as well as PET scan, etc. along with the datasets of BraTS as in Menze et al. (2014), Cocosco et al. (1997), as well as in 
BrainWeb (http://www.bic.mni.mcgill.ca/brainweb/). Images preprocessing contains separate strategies for digitising images, reduction of noise, image up gradation and enhancement. Similarly, distinct strategies are utilised for separation as well as isolation of target regions also free computing tools for segmenting images are accessible in Neuroimaging Tools \& Resources Collaboratory (https:/www.nitrc.org/). Statistics and overall features are dug out in parts of extracting features. At last, diverse sorts for artificial intelligence prototypes are used for the grouping or bunching those influenced and uninfluenced parts of the human cerebrum or brain and yield image for a consultation to a medical expert and a settling medical conclusion.

Figure 1 (a) MRI (b) CT and (c) PET scans (see online version for colours)

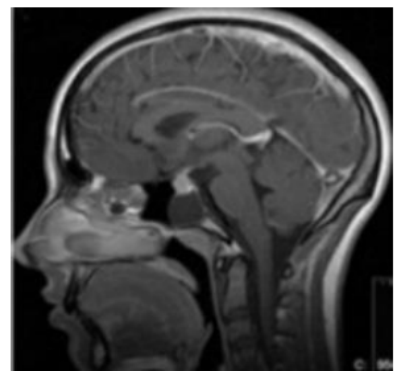

(a)

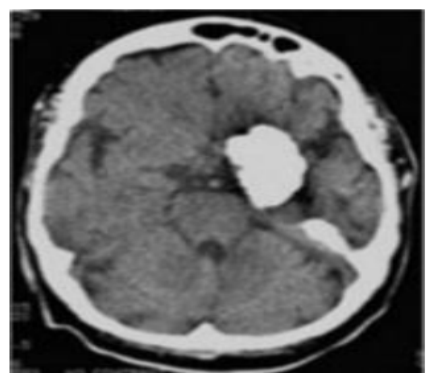

(b)

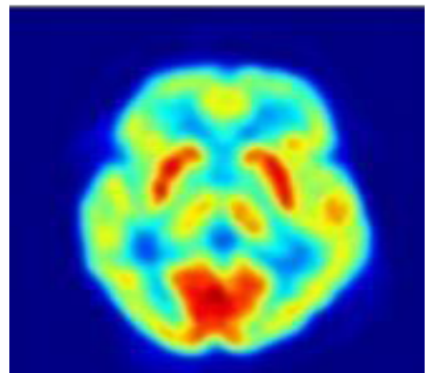

(c)

Figure 2 Automated BT detection flow

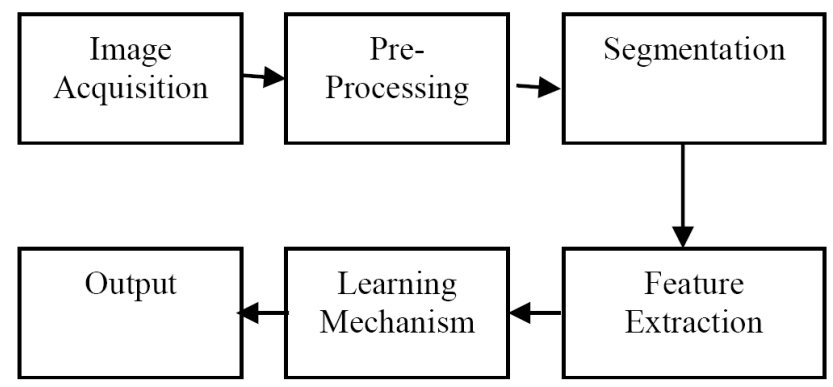

Presented paper is giving an ample and swift analysis of the most recent techniques for BT analysis, grouping and segmentation. The work centres upon abstract analysis on the types of categorisation methods and assessment of extracted features. An enormous quantity of time and memory is required as an image requires immense data for representation. In order to minimise the usage of memory, data and time features are extracted from images that in turn contain useful information's. This paper explores diverse techniques for segmentation of the BT which will lead to:

- $\quad$ quick diagnosis

- $\quad$ high accuracy of results

- $\quad$ less time to detect

- $\quad$ help early stage identification of disease by medicinal experts 
- $\quad$ saves life and time.

Features types that can be extracted from an image can be classified as in Figure 3.

Figure 3 Types of features that can be extracted using MRI

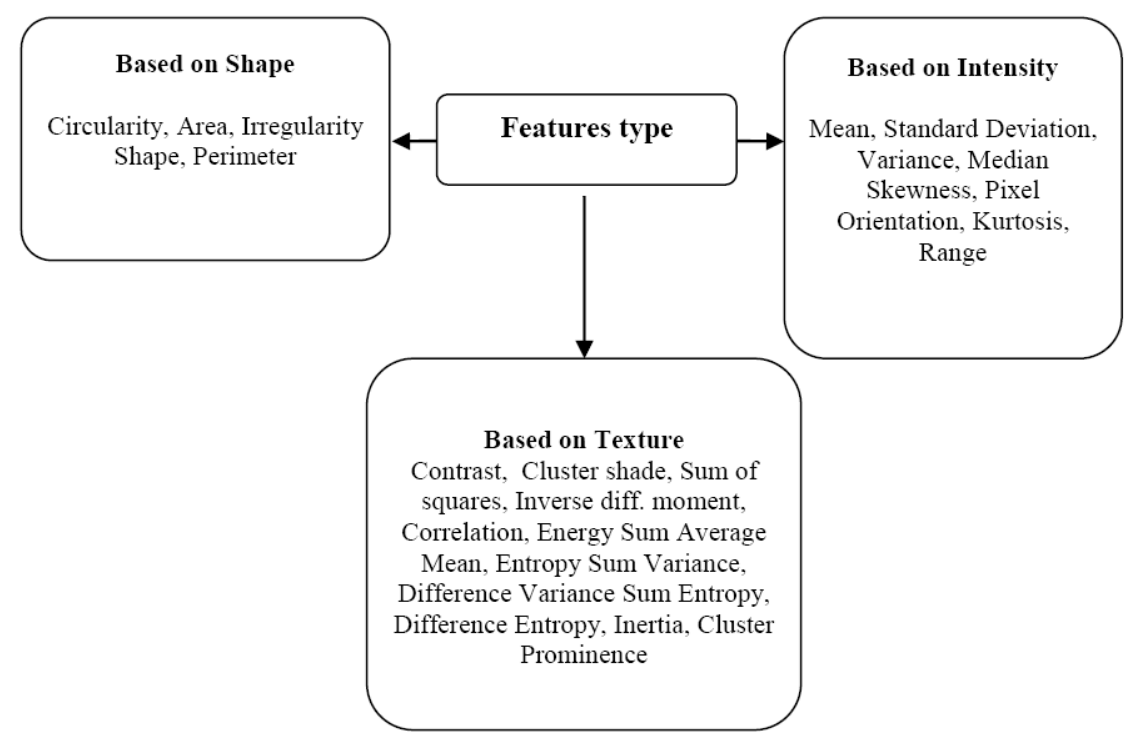

\section{Feature extraction methods overview}

Feature extraction is a dimensionality reduction process where the original raw data is converted into more convenient sets for processing. A huge amount of variables is an attribute of such huge datasets where numerous computations are required to process. Extraction of features is basically a process which picks or/and merges variables thereby efficiently plummeting the extent of data to be processed, although maintaining the originality of the initial dataset. Some basic feature extraction techniques are follows.

\subsection{Haar features}

Haar features are digital images features used in object recognition. It owes its name to their intuitive similarity with Haar wavelets. It performs the calculations in real time by summing the white region pixels and subtracting the sum of black region pixels from it. In this, a large number of weak classifiers are combined to be a strong classifier in order to make decisions based on Haar features. As per Lienhart and Maydt (2002), it uses integral images, therefore, it is very simple and fast to calculate.

\subsection{Histogram of oriented gradients}

As reported in Lowe (2004), histogram of oriented gradient (HOG) is used to calculate the gradient magnitude and orientation at each pixel of an image. It divides the orientations of the pixels into $N$ bins, e.g., 360 degrees can be divided into 18 bins, the 
gradient magnitude of each pixel is then voted into the bin corresponding to its orientation. Some of its improved variants are:

- Divide the image into cells, compute HOG for each cell and concatenate them.

- Use a Gaussian window to weight the gradient magnitudes.

- $\quad$ Normalise the final HOG to unit magnitude.

\subsection{Local binary patterns}

Linear binary pattern (LBP) utilises a $3 \times 3$ matrix and compare eight-connected neighbourhood with the centre pixel. If the neighbourhood pixel value is found to be greater the centre pixel value it is replaced with ' 1 ' else ' 0 '. Then, a binary number constructed by going clockwise as shown in Figure 4. The centre pixel is then replaced with the decimal value of the binary number (Silva et al., 2015). The binary number is sensitive to the starting point and therefore LBP is not rotation invariant which is one of its disadvantages.

Figure 4 Binary numbers from decimal in LBP

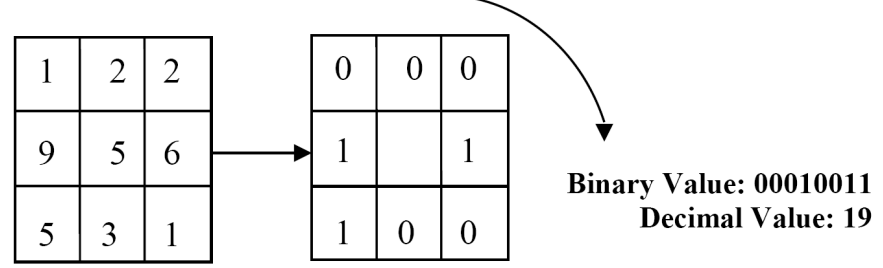

\subsection{Harris corners}

As per Sánchez et al. (2018), a corner is a point around which the gradient has two or more dominant directions. Corners can be repeatedly detected under varying illumination and viewpoint changes. In 1988, Chris Harris and Mike Stephens introduced this as an enhancement of Moravec's corner detector. Without loss of generality, we will assume a greyscale two-dimensional image is used. Let this image be given by $I$. Consider taking an image patch over the area $(x, y)$ and shifting it by $(\Delta x, \Delta y)$. The sum of squared differences (SSD) between these two patches, denoted $f$, is given by:

$$
f_{\left(x_{k}, y_{k}\right)}=\sum_{\left(x_{k}, y_{k}\right) \in W}\left(I\left(x_{k}, y_{k}\right)-I\left(x_{k}+\Delta x, y_{k}+\Delta y\right)\right)^{2}
$$

In equation (1), I( $\left.x_{k}+\Delta x, y_{k}+\Delta y\right)$ can be approximated by a Taylor expansion and equation can be written as:

$$
f_{\left(x_{k}, y_{k}\right)} \cong \sum_{\left(x_{k}, y_{k}\right) \in W}\left(I_{x}(x, y) \Delta x-I_{y}(x, y) \Delta y\right)^{2}
$$

Equation (2) may be expressed in matrix form as: 


$$
f_{\left(x_{k}, y_{k}\right)} \approx(\Delta x, \Delta y) M\left(\begin{array}{l}
\Delta x \\
\Delta y
\end{array}\right)
$$

where $M$ represents the structure tensor or second-moment matrix.

\subsection{Scale invariant feature transform}

Harris corner detector is rotation-invariant, which means, even if the image is rotated, we can find the same corners. It is obvious because corners remain corners in the rotated image also. But what about scaling? As per Lindeberg (2014), a corner may not be a corner if the image is scaled. A corner in a small image within a small window is flat when it is zoomed in the same window. So, Harris corner is not scale invariant. In 2004, David Lowe, of UBC, proposed a new algorithm, scale invariant feature transform (SIFT), which extract key points and compute its descriptors. There are mainly four steps involved in the SIFT algorithm, as:

- $\quad$ scale-space extrema detection

- $\quad$ key point localisation

- orientation assignment

- $\quad$ key point descriptor.

SIFT feature extractor has following properties:

- $\quad$ repeatable key points

- scale invariant

- rotation invariant

- $\quad$ robust to viewpoint

- robust to illumination changes.

\section{BT detection techniques}

Ample of analysis and apprehension of BT by MRI had been proposed by scholars. Here, we present an analysis of some current and most recent works. As per Joseph et al. (2014), for miss clustered BT proposed morphological filtering and K-means clustering. Bhosale et al. (2017) suggested that for MRI images of BT, fuzzy C-means (FCMs) and $\mathrm{K}$-means can be utilised and thereafter support vector mechanism (SVM) model is used for classification of the patient stage of the tumour using MRI analysis of BT and again classified the data of the accommodating application in an SVM. Dahab et al. (2012) altered the calculation for careful edge identification to identify forms of BT and probabilistic simulated neural networks (PNN) is then applied in view of taking in learning vector quantisation (LVQ) arrangement for BT by looking into MRI scans. The test and training set which comprises 16 and 64 images, respectively attained cent percent accurate results. They additionally guaranteed that those altered PNN supported by LVQ framework diminished processing time by nearly $79 \%$ in contrast with dated PNN. Mustaqeem and Javed (2012) analysed the MRI scans of 30 male and females in the age 
group of 26 years. The scan is then transformed into greyscale. Separate filters are utilised for the reduction of noise and the enhancement of scanned images employing separate high pass filters. The image is then converted to binary form after applying segmentation, thresholding and watershed methods for recognising BT by implementing various morphologic operations. Rajesh and Patil (2013) additionally utilised high pass filter for gain enhancement and median filtering for removal of noise from images. Finally, that division and extraction of BT utilising morphologic operation and Meyer's flooding watershed for BT. MRI scans are also utilised.

Selkar and Thakare (2014) primarily utilised MRI scans. The scans were changed over under greyscale and then enhancing of those scans and enrichment techniques were applied. Thereafter, histogram method of threshold segmentation is applied for achieving the target outcome. Then, boundary extraction of limit is done utilising identification of edge techniques. Finally, shape and span of the BT found utilising scientific operations. Laddha and Ladhake (2014) exhibited different preprocessing strategies for evacuation for noise removal, texture up gradation of scanned images and then enriching those scans. Thresholding and watershed segmentation is applied in order to improve various features of a scanned image. Finally, morphologic operators will shrink and dilate the picture for identification of BT. Subramanyam and Raviraj (2015) implemented identification of a BT into four phases. In the primary phase, $3 \times 3$ median filter is used for noise removal. In the subsequent step, substantial elements are found out utilising shapes, intensities, texture, and so forth. Then afterward, feature extraction is implemented so as to process easily the small segments of the image to find the actual affected BT area. Finally, post-processing will be carried toward diverse operations such as figuring those high-intensity regions. For superlative outcomes sensitivity, mean and variance are calculated. Ghare et al. (2015) confirmed the BT existence by applying FCM. The projected arrangement has eight stages. Initially, ascribe an MRI scan, additional its greyscale transformation is done. In the next stage, thresholding is activated to a binary image from a grey image. The succeeding step involves Sobel edge for extracting area of the skull through distinct operations. The next phase includes, soft clustering method of FCM is employed. It separates the components into C-fuzzy groups. The next stage includes measuring image white points. In the next phase, it is calculated that which part is infected by BT and in the last step resulted in afflicted regions of the BT is shown.

Mohan et al. (2015) uprooted noisy portion of the image for its up gradation further converted and disintegrated it by wavelet channel. Such deteriorated scanned pictures are then combined by their coefficients. In the next step, fused images are then segmented. As intensity and threshold segmentation is a smooth process. For the extraction of BT, feedforward technique of neural network is used. An additional skull stripping system is utilised which collects information about those parts of the brain which is not normal. Naik and Patel (2013) additionally implemented a median filter, noise eradication mechanism and morphological operator along with a power-law transformation in the processing phase. The histogram and features based on textural information are then extracted and given to a decision pyramid to introduce the approach which is focused upon segmentation strategies. BT detection utilising histogram is utilised and furthermore, for upgrading outcomes of segmentation, optimised fuzzy logic based on FCM clustering is applied. To arrange neighbour pixel in the spatial domain for calculating statistics, membership functions are used. Angoth et al. (2013) done MRI along with CT scan for examining those volumes and area of the BT. After that, wavelet 
analysis is performed using low and high-frequency decomposition. Then, the result is compared with the segmentation algorithm and GVF. Hence, they resolved that the algorithm suggested provides for superior effects over other calculations.

Patil et al. (2016) done the recognition of a BT in the noisy clinical image and the images are then combined. On those fused images which are in binary form, segmentation and image enrichment is done. For decomposition, wavelet transforms by parameter modification is used. Thus, the BT is distinguished through the pixel intensity. Deorankar and Salam (2015) introduced four ways for the identification of the BT. In the first step, Gaussian filter is used to remove the noise. In the next stage, the histogram technique is used for segmentation based on colour for pointing out crust and turfs in the image. In the third step, extraction of features is done by deriving abnormal features of the image and thereafter identifying pixels features by applying a threshold. Finally, estimation or arrangement incorporates characterising the impostor utilising dilation and shape comparison with aged BT using astrocytoma. After and before results are compared using fuzzy expert systems.

Padmavathi and Megala (2015) performed the spatial sifting over the pixels of a scanned image. In the subsequent stage, segmentation is done by segregating the areas under associated parts of the image hence analysing each area. Segmentation is used for identification of BT location and thereafter thresholding and edge detection with clustering is applied. Kalaiselvi et al. (2016) utilised MRI pictures. Noise is removed during preprocessing step by median filter and bottom-hat, top-hat, Otsu's segmentation, and minima transformation are done for image enhancement. The fuzzy symmetric measure is applied for detecting an abnormality. When the brain's abnormal part is identified, Otsu's strategy for thresholding is employed to differentiate the anomalous part from the image for its background separation. True and false rate parameters are used to check the performance of all the process. Logeswari and Karnan (2010) introduced a procedure for identification of BT where a greyscale image input of 256 $\times 256$ is taken in JPEG. To remove noise from the image, its preprocessing is done using a weighted median filter. Segmentation of an image is done using artificial neural network (ANN) self-organising map (SOM) and HSOM. SOM is utilised for training and mapping whereas HSOM is utilised for the enrichment of the graphical map. Thereafter, the after-effects of BT are measured with cells and pixels of tumour of the measurement $3 \times 3,5 \times 5,7 \times 7,9 \times 9$, and $11 \times 11$ windows.

Handore and Patil (2010) performed a test on 42 MRI pictures of BT. MRI scans are changed into the greyscale of size $256 \times 256$. In the next step after noise removal, power law transformation and the median filter is employed for image enhancement. To detect the main region of BT segmentation is used. The histogram is used for segmentation of threshold values, minimising T1 and maximising T2. Features of pixels are calculated by cropping. Azhari et al. (2014) utilised MRI as well as CT images as input and median filter for image enhancement. Outcomes are derived by K-mean segmentation and clustering and enhancing the morphologic operator's maxima. A novel device is used for segmentation of image which lessens the time taken in processing and augments precision of solving the problem by reducing aberrant noise and activated distinct filters for sharpening of an image and its enrichment. An algorithm called canny edge detection is used in order to find an edge in the image that identifies the tumour edges. The histogram is used for obtaining grey-level frequencies and grouping of identical neighbours. Addition and subtraction are done by using morphological operators for the vectors that target for maxima enhancement. 
Mallikarjuna (2015) detected tumour by using the normalised histogram. At first, MRI scan which is taken as an input is enhanced through reduction of noise and interruption by median and spatial filtering. The image is converted into a histogram and the result will have an image containing BT. To sum the probability, the normalised histogram is developed and judgement is derived from the probabilities that either tumour exist or not. Nisar et al. (2015) used semi and fully programmed segmentation and manual method to introduce multi modularity. The conventional neural network is utilised for deep learning. Those primary targets are to upgrade the outcomes of magnetic resonance spectroscopy (MRS) and diffusion tensor imaging (DTI) PET. In Chandra and Rao (2016), for segmentation purpose, projected a genetic algorithm (GA). For ciphering of best and the minimum chromosomes, the fitness function is acclimated and demarcated as $f=1 / M$. Attained targeted SVR in ranges 20 to 44 and segmentation accurateness of $82 \%-97 \%$. Abdel-Maksoud et al. (2015) functioned an algorithm of image segmentation through relating them in time accuracy and performance. In order to decrease the processing time, brain surface extractor algorithm is used for identifying the brain surface using KIFCM algorithm with hybrid clustering. Işın et al. (2016) developed semi-automatic segmenting of tumour and identification of dead cells through MRI scans. Primarily, the MRI scans are changed into digital form and thereafter snake contour techniques are used along with density assessment using adaptive segmentation.

Damodharan and Raghavan (2015) worked on brain complexity such as RF coil homogeneity, systematic conflicts, and fragility of brain tissue which are considered to be intricate after segmentation. The skull striping marks the start of the systems process that includes thresholding binarisation, morphologic operations, and a binary mask is employed. An orthogonal polynomial is used along white matter (WM), grey matter (GM), and cerebrospinal liquid (CSF) segmenting. Mean, entropy, variance, and wavelet-based statistical features are used. Neural network of feedforward type is used. Network contains three layers as input, hidden and output involving 25, 5, 1 neurons, respectively. Feedforward network along with multilayer PNN is utilised to make the associations neurons. Relative learning is done by k-nearest neighbour (k-NN) and Bayesian classification. Suggested neural network classifier transformed preferred effects over others. Nabizadeh and Kubat (2015) suggested a technique which consists of 2D single spectral anatomical about MRI scans. Identification of BT depends upon histogram of two mutual brain information. Diversified characteristics like normal contrast, mean, Gabor, entropy and energy, grey-level co-occurrence matrix (GLCM), skewness kurtosis along with the grey-level run period for extracting BT. HoG and LBP are employed. Aggregation is used to calculate the performance mathematically.

Nakhmani et al. (2014) utilised FCM, K-means, as well as thresholding for the purpose of segmentation and Otsu for binarisation. For classification, ANN framework is used. Subashini and Sahoo (2013) used BRATS dataset for investigating segmentation of BT. For the purpose of classification, convolution neural network $(\mathrm{CNN})$ is executed. Using BRATS online evaluation assessment is done. Sugapriya (2017), for distinctive datasets of BT, suggested an algorithm for BT segmentation. During the primary step filtering and resizing is applied and thereafter original image is converted to binary form using Otsu method and thresholding is done. In this microcontroller of PIC type is utilised and performance analysis is based on PBM, DB and IFV. Havaei et al. (2016) analyse the brain MRI along with hybrid machine learning using CAD for the identification of BT. These extracted features are used for reducing the wavelet 
coefficients through examination and to detect the anomalous and usual brain cells. Back propagation $\mathrm{NN}$ is used for analysing BT dataset of over 100 images and around 99\% accuracy is obtained.

Mustafa and Hassan (2016) sort the BT images based on their types and grades using SVM. Glioma, normal, meningioma, metastasis along with four grades of astrocytoma are here focused upon in this paper. In this paper, first and features of second order along with their combination are utilised by SVM classifier. Outcomes indicate that the features engaged by a second order for types of tumour and its grade acquired a precision of $85 \%$ and $78.26 \%$ proportionately. First order features have a precision of $65.5 \%$ and $62.31 \%$ at the same time individually. However, when they are used simultaneously precision of $84.48 \%$ and $68.1 \%$ is achieved. The outcomes indicated that SVM meets expectations satisfactorily in BT sort's classification but grades of the tumour are not satisfactorily identified. Hasan and Ahmad (2018) used two-level authentication systems in order to detect the tumour. He used an algorithm called watershed for segmenting the region containing the tumour. For comparing the segmented image with the original, one scale invariant Fourier transform is used. Authors also calculated the tumour area by counting the total numbers of white pixels in the MRI scan. Shree and Kumar (2018) targets to enhance the identification of an infected area of BT. They focused upon the noise removal along with GLCM and discrete wavelet transform (DWT)-based feature extraction. DWT is used for wavelet coefficient extraction which localises frequency information of signals which are important for classification. For texture feature extraction, GLCM is used which differentiates normal and abnormal tissues in the brain. They also used PNN (FNN) classifiers to train and test the precision in detection of tumour in the brain.

Nagarajan et al. (2016) presents a hybrid image retrieval system by GA. They developed the system in three steps, in the first three different algo's are utilised to extract the important features of an image. In the next phase, potential features are identified using the 'branch and bound' algorithm as well as artificial bee colony algorithm. Islam and Zhang (2018) have presented a deep convolutional neural network (CNN) technique for the brain MRI data analysis for the identification of different stages of the disease and for early-stage diagnosis of disease. They used a small dataset to train the network but still able to get a superior outcome even with the imbalanced dataset. Khalil et al. (2018) employed three types of techniques for extracting features: GLCM, LBP and HoG. The result of each is then passed through the k-NN classifier and the subsequent difference in the values of the classifier is then combined by a fusion operator to enhance the accuracy of classification. Gopal and Karnan (2010) used FCM along with GA and PSO for image clustering. Preprocessing and enrichment of an image are followed by segmentation and classification for circling out the suspicious region of background tissues. The time taken for execution with FCM and GA is 93.39 secs while for PSO and FCM it is 100.3 secs. The accuracy for FCM with GA was $74.6 \%$ whereas for FCM and PSO, it was $92.3 \%$ with an error of 0.3919 and 0.1273 for each of the techniques.

Islam et al. (2013), in his paper, presented a modified AdaBoost classifier for mild and composite BT classification. In modified AdaBoostalgo, assigning of weights is based on the ability of the classifier to categorise the challenging samples and sureness in such categorisation. The system effectiveness is verified by the results of 14 patients with more than 300 MRI scans. Kharrat et al. (2010) proposed a hybrid methodology for categorisation of tissues in the brain. This is based on SVM and GA where the extracted 
features are texture-based features derived by spatial grey-level dependence method (SGLDM). The features thus extracted are fed to SVM as input and GA is used to solve the feature choice classification problem. The presented method has an accuracy minimum of $94.44 \%$ to a maximum of $98.14 \%$. Anitha and Murugavalli (2016) presented an adaptive K-mean procedure for two-tier classification and segmentation. In the presented method, feature extraction is done using DWT and is trained using self-organising NN and k-NN. The two-tier categorisation system classifies BT in the dual training method. Normal and abnormal MRI scans are identified by utilising the technique of segmentation. The MATLAB R2013a implemented system gives improved total performance and accuracy when compared to other orthodox procedures.

Subashini et al. (2016) projected a method that does not involve the introduction of instruments into the body for locating the BT utilising MRI scans. It consists of the processing of an image, followed by segmentation, BT isolation, extraction of image and categorisation. Performance evaluation is carried out based upon elapsed time, accuracy and efficiency. PCNN is utilised for the removal of noise. Among various available techniques, naive classifier provides enhanced results than LVQ and SVM whereas FCM produces enhanced outcomes. In their work, they had used 164 images for the purpose of training the system and 36 for the testing of the system out of 2,000 total MRI scans. Their presented method gives a precision of $91 \%$ and takes minimal time for tumour recognition. Zulpe and Pawar (2012) utilised 80 MR pictures. They focused their efforts to arrange four distinctive tumour classes, for example, carcinoma, meningioma, astrocytoma, sarcoma and metastatic bronchogenic. Their characteristic extraction has been carried utilising GLCM that concentrated on textural characteristics extraction. For categorisation, a double level neural network feedforward type is utilised. It utilised Levenberg Marquart (LM) nonlinear optimisation algorithm that provides a precision rate of about $97.5 \%$. A database for $101 \mathrm{MR}$ pictures is utilised by Amulya and Prathibha (2016) and arranged the images as BT and non-BT imageries. Characteristics of SURF were utilised to extricate pictures from the information set. These concentrated features were provided for with k-NN classifier. The author in the presented paper extracted features using SIFT and SURF methods. The output was checked based on exactness, the time elapsed, specificity and sensitivity. Exactness claimed by SURF is $94.3 \%$ and SURF-SIFT features are $96.2 \%$ individually. SURF and SURF-SIFT offers around $97 \%$ and $96 \%$ sensitivity and $81.96 \%$ and $85.71 \%$ preciseness, respectively. They have an elapsed time of $14.6 \mathrm{sec}$ and $1,935.76 \mathrm{sec}$, respectively. Both the characteristics have an exactness of $96.22 \%$ utilising k-NN classifier.

Vidyarthi and Mittal (2015) uses 150 MRI pictures among which 53\% pictures were of males and $47 \%$ pictures were of the female. Harmful tumours, for example, glioblastoma multiforme (GBM), central neurocytoma, gliomas, intra ventricular threatening impostor and metastasis were displayed in the dataset. Gabor wavelet and discrete wavelet transformation were used for obtaining 60 features form the dataset. k-NN, multi-level SVM and BPNN were used for the classification. Cumulative variance method (CVM), GA, and independent component analysis (ICA) were utilised for feature extraction. Different classifiers with different extraction features were tried. The suggested feature determination algorithm CVM provides higher precision with all classifier for harmful tumour of a high order. The most excellent outcome has been attained with exactness for $97 \%$ to the blending of Gabor-wavelet + CVM + BPNN. Demirhan et al. (2015) propose a segmentation algorithm that divides MR pictures of 
brain under WM, tumour, GM, oedema, and CSF. The statistics utilised has T1, T2, and FLAIR images of 20 persons. The suggested algorithm stripes-off skull prior to segmentation. The divisions were performed using SOM. The calculation is done utilising the unsupervised learning algorithm which is fine-tuned with LVQ. The suggested technique needs segmentation exactness for $100 \%$ to CSF. The intended segmentation correctness of WM with GM is $91 \%$ and $87 \%$.

ParthaSarathi and Ansari (2017) displays recovery structure for digging out comparative 3D BT in MR brain volumes in reply to tumour volume in the examination. Comparable volumes will relate closeness on the spatial area of the brain structures. The portion under examination pertains to a fresh tumour volume and the resulting slices have a place with the tumour volumes identified with past histories stored in the database. A database of BT volumes identified with patients will be structured. Information of the schema will be an inquiry volume correlated with a fresh case. For the recovery trials, T1 post-contrast axial scans are utilised. In order to assimilate all the images initially, an algorithm has been devised, such that the imagery is acceptable for advance processing. A quick, authentic and dependable algorithm is activated on the already processed images for 'seed' detection. The database comprised of 12 tumour volumes (GBM) that were utilised for recovery purpose. For performing the experiments, 2D multi-slice representational of $3 \mathrm{D}$ volumes were utilised. T1 post-contrast scans of the axial slice with the thickness of about $6 \mathrm{~mm}$ and in between slice width of $6.5 \mathrm{~mm}$ were utilised individually. Each volume consists of 24 slices. The observation was carried upon 12 brain images of GBM retrieved from axial T1 post-contrast scans between the case under examination and the stored tumour volume in the database. Tumour seed identification algorithm attained a seed accuracy of about over $90.7 \%$ which is huge.

Bahadure et al. (2017) suggested a calculation to perform segmentation, extraction of desirable features, and categorisation of perception of human vision, which distinguishes different objects, diverse textures, contrast, brightness, and depth of a picture. They utilised a consolidation of naturally propelled Berkeley wavelet change (BWT) and SVM for classification for augmenting exactness of diagnosis. They utilised two benchmark datasets and case dataset gathered from professional radiologists, which incorporated 15 sample images of patients having nine slices for each patient. For the reason of investigation, $22 \mathrm{MR}$ images from digital imaging and communications in medicine (DICOM) dataset were taken; every image incorporated was of tumour-infected brain tissues. However, this dataset did not have what was desired. BrainWeb dataset was the next dataset considered, which comprises of complete $3 \mathrm{D}$ recreated MR information retrieved from T1, T2-weighted MRI, and proton density-weighted MRI. This dataset incorporated a range of slice thicknesses, levels of noise, and non-uniform intensity levels. Mostly T2-weighted modality with a thickness of $1 \mathrm{~mm}$, the noise of $3 \%$, and non-uniform intensity of $20 \%$ was utilised. Out of 44 images, 13 brain tissues are infected with the tumour. The most recent dataset gathered from professional radiologists with all modalities comprised of 135 pictures from 15 patients. This dataset had practical images which aided in evaluating the outcomes of our technique with the manual investigation of radiologists. For adaptive fuzzy inference system (ANFIS), it came approximately $86.14 \%$, for back propagation it is $80.29 \%$, and for SVM it is $90.54 \%$ whereas for $\mathrm{k}-\mathrm{NN}$ it is $84.55 \%$ without feature extraction. 
Feature extraction for brain tumour analysis and classification

Table 1 Types of BT and grade classification related work

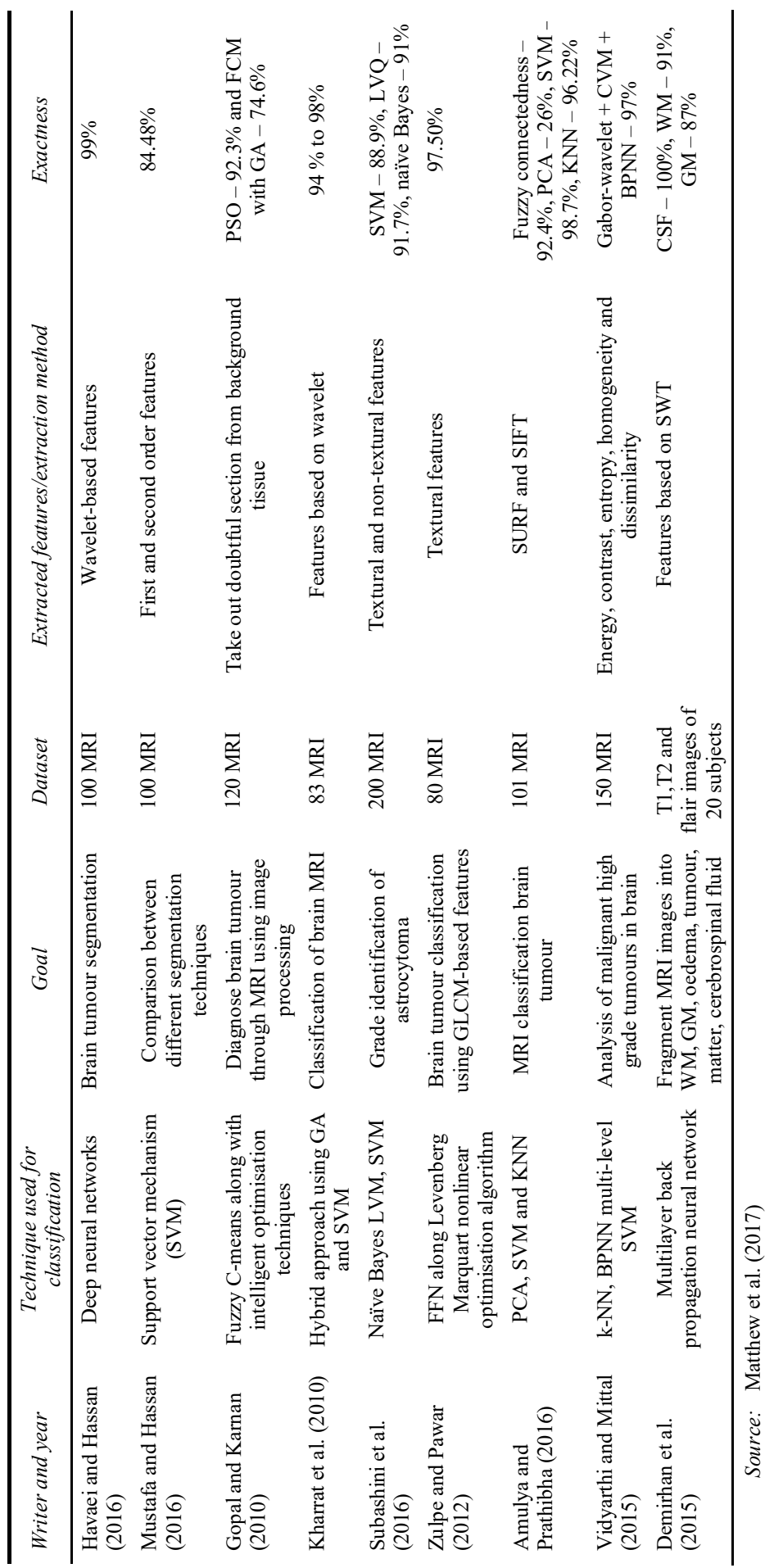


Table 1 Types of BT and grade classification related work (continued)

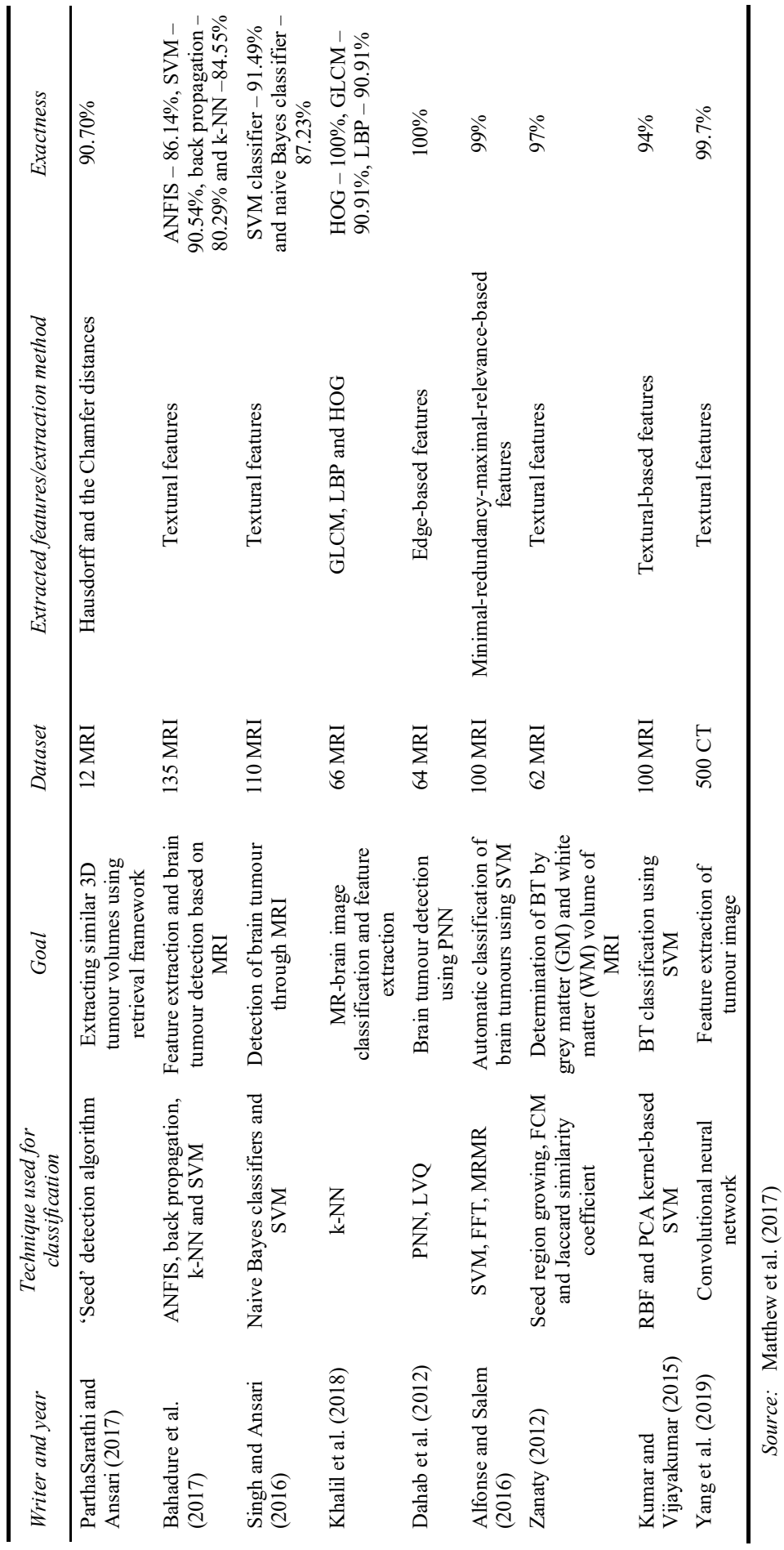


Figure 5 Comparison of different classification techniques (see online version for colours)

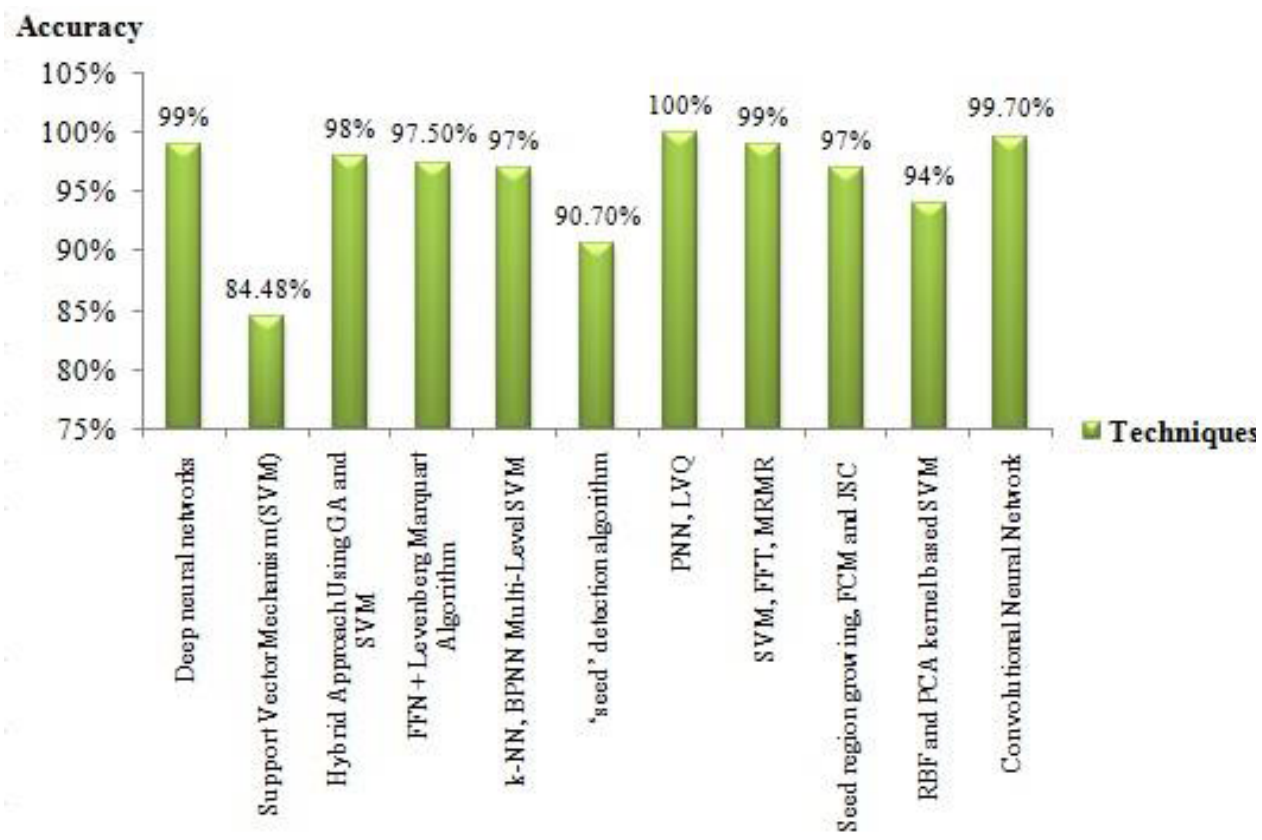

Singh and Ansari (2016) suggested a novel method which incorporates histogram normalisation along with K-means segmentation. Preprocessing of the input image is done so as to uproot the redundant noise. The normalised histogram MR images are classified using SVM and naive Bayes classifiers. Lastly, in order to point out the tumour from the MRI, the image is segmented using a K-means algorithm. Effectiveness of classifiers is found out to be: $87.23 \%$ for naive Bayes classifiers and $91.49 \%$ for SVM classifier. The tumour infected image is then segmented using K-means clustering. Alfonse and Salem (2016) have introduced a method for BT classification by using support vector machine. FFT is utilised for extracting features and enhancing the exactness of the classifier and feature reduction is done by minimal-redundancy-maximal-relevance (MRMR) technique. This strategy needs to acquire a precision of about 98.9\%. Zanaty (2012) suggested a technique for segmentation of BT using an amalgamation of seed region growing, FCM and Jaccard similarity coefficient algorithm, to calculate the segmentation of white as well as GM in MRI. This technique acquired $90 \%$ in average segmentation at $3 \%$ and $4 \%$ noise level. Kumar and Vijayakumar (2015) acquainted principal component analysis (PCA) and radial basis function (RBF) kernel-based SVM for BT segmentation and classification and asserts comparability of $96.20 \%, 95 \%$ overlap fraction, $0.025 \%$ of the extra fraction. The precision for identifying types of BT is $94 \%$ with $7.5 \%$ of error. Table 1 representing the types of BT and their grade classification related work is given.

Yang et al. (2019) takes 500 CT images of the tumour as the object of research, and initially carry out the LBP for extracting features of CT images using rotation invariance. With the shift and change in rotations, the CT images remained stationary with respect to the coordinate framework. Their proposed strategy precisely depicted the textural features of the image's shallow layer of the tumour, and hence augmenting the 
healthiness in the depiction of the image region. Focusing on CNN-based extraction of image features; the fundamental structure of CNN is built. To overcome the restrictions of human and machine vision, they extended their research to multi-channel input $\mathrm{CNN}$ for extracting features from an image. In order to improve the exactness of CNN algorithm two models, Dense Net and Xception are constructed. From their investigational outcomes, it is evident that the CNN exhibits greater exactness $(99.7 \%)$ in tumour image feature extraction. They also compared $\mathrm{CNN}$ with various standard algorithms in LBP mode.

Table 1 exemplifies the types of BT and grade classification related work and Figure 5 presents the comparisons of some major classification techniques.

\section{Possibilities of computerised MRI detection}

Computerised systems act as an interface between the human and machine. It combines recognition, collection, representation, creation, organisation, communication, transformation, evaluation and control of information along with visualisation into one system which should be more effectively utilised. El-Dahshan et al. (2014) states that computerised system can improve the investigative capabilities of a general medical practitioner and hence minimise the time taken for precise analysis. The aim should be to present a comprehensive computerised system that works equally well no matter what the quality of image and its size is. Therefore, computerised system remains an open-ended issue. Many future prospects that may develop a better computerised system for BT imaging are:

1 Database acquirement from diverse organisations with different quality of images for medical assessment and upgrading the computerised system.

2 Advancing the exactness of classification by resourceful features extraction and vast dataset for training.

3 Making the most of different techniques of machine learning and amalgamate them into a single system.

\section{Conclusions}

The works of literature presented here have shown that some of the methods are discovered only for the purpose of segmentation; while few of them were only to extract features from the image and its classification. In most the above-discussed papers, extraction of features and feature vectors reduction for effectual segmentation of CSF, $\mathrm{WM}, \mathrm{GM}$ as well as BT contaminated region and analysis on combined approach is not dealt with. Additionally, in some cases, extracted features are less and consequently extremely low precision in BT detection has been achieved. Moreover, the overlap computation which plays a pivotal role in judging the precision of BT segmentation is not seen. Improvement in segmentation and identification of BT by MRI images precisely and in a way in which it can be reproduced is a challenge in the biomedical imaging field. Although, incredible possibilities are there for computerised systems in order to segment and identify BT but a lot needs to be done yet. For the purpose to save human life, we 
need to explore more imaging techniques to deal with this grave problem and to aid medical experts for timely diagnosis of diseases.

\section{References}

Abdel-Maksoud, E., Elmogy, M. and Al-Awadi, R. (2015) 'Brain tumor segmentation based on a hybrid clustering technique', Egypt. Informatics J., Vol. 16, No. 1, pp.71-81.

Alfonse, M. and Salem, A.B.M. (2016) 'An automatic classification of brain tumors through MRI using support vector machine', Egyptian Computer Science Journal, September, Vol. 40, No. 3, pp.11-21.

American Cancer Society (2016) About Brain and Spinal Cord Tumors in Adults, pp.1-17.

Amulya, C. and Prathibha, G. (2016) 'MRI brain tumor classification using SURF and SIFT features', International Journal for Modern Trends in Science and Technology, July, Vol. 2, No. 7, pp.123-127, ISSN: 2455-3778.

Angoth, V., Dwith, C.Y.N. and Singh, A. (2013) 'A novel wavelet based image fusion for brain tumor detection', Int. J. Comput. Vis. Signal Process., Vol. 2, No. 1, pp.1-7.

Anitha, V. and Murugavalli, S. (2016) 'Brain tumor classification using two-tier classifier with adaptive segmentation technique', IET Journals, Vol. 10, No. 1, pp.9-17.

Azhari, E-E.M., Hatta, M.H., Htike, Z.Z. and Win, S.L. (2014) 'Glioblastoma multiforme identification from medical imaging using computer vision', International Journal of Soft Computing, Mathematics and Control (IJSCMC), May, Vol. 3, No. 2, pp.1-12.

Bahadure, N.B., Ray, A.K. and Thethi, H.P. (2017) 'Image analysis for MRI based brain tumor detection and feature extraction using biologically inspired BWT and SVM', International Journal of Biomedical Imaging, Vol. 2017, 12 pp., Article ID: 9749108 [online] https://doi. org/10.1155/2017/9749108.

Bhosale, P., Lalge, P., Dhandekar, A., Gaykar, P. and Pate, P.V. (2017) 'Brain tumor detection and segmentation', Int. J. Innov. Res. Sci. Eng. Technol., Vol. 1, No. 2, pp.1-8.

BrainWeb, BrainWeb: Simulated Brain Database [online] http://www.bic.mni.mcgill.ca/brainweb/ (accessed 05/01/2019).

Chandra, G.R. and Rao, K.R.H. (2016) 'Tumor detection in brain using genetic algorithm', Procedia Computer Sci., Vol. 79, pp.449-457.

Cocosco, C.A., Kollokian, V., Kwan, R.K-S., Pike, G.B. and Evans, A.C. (1997) Brainweb: Online Interface to a $3 D$ MRI Simulated Brain Database in NeuroImage.

Dahab, D.A., Ghoniemy, S.S.A. and Selim, G.M. (2012) 'Automated brain tumor detection and identification using image processing and probabilistic neural network techniques', Int. J. Image Process. Vis. Commun., Vol. 1, No. 2, pp.1-8.

Damodharan, S. and Raghavan, D. (2015) 'Combining tissue segmentation and neural network for brain tumor detection', Int. Arab J. Inf., Vol. 12, No. 1, pp.42-52.

Demirhan, A., Toru, M. and Guler, I. (2015) 'Segmentation of tumor and edema along with healthy tissues of brain using wavelets and neural networks', IEEE Journal of Biomedical and Health Informatics, July, Vol. 19, No. 4, pp.1451-1458.

Deorankar, A.V. and Salam, A.K.A. (2015) 'Systematic approach for brain tumor detection using rough sets on DICOM images', Int. J. Res. Appl. Sci. Eng. Technol., Vol. 3, No. 3, pp.645-650.

El-Dahshan, E-S.A., Mohsen, H.M., Revett, K. and Salem, A-B.M. (2014) 'Computer-aided diagnosis of human brain tumor through MRI: a survey and a new algorithm', International Journal of Expert Systems with Applications, 1 September, Vol. 41, No. 11, pp.5526-5545.

Ghare, M.N.S., Gaikwad, N. and Kulkarni, N. (2015) 'Detection of possibility of brain tumor using image segmentation', Int. J. Innov. Res. Comput. Commun. Eng., Vol. 3, No. 4, pp.1-7. 
Gopal, N.N. and Karnan, M. (2010) Diagnose Brain Tumor Through MRI Using Image Processing Clustering Algorithms Such as Fuzzy C Means Along with Intelligent Optimization Techniques, IEEE.

Handore, S.S.V. and Patil, P.M. (2010) 'Brain tumor segmentation from MRI using GLCM', Int. J. Emerg.Technol. Eng., Vol. 2, No. 4, pp.591-595.

Hasan, S.M.K. and Ahmad, M. (2018) 'Two-step verification of brain tumor segmentation using watershed-matching algorithm', Int. J. on Brain Informatics, Vol. 5, No. 8, https://doi.org/10.1186/s40708-018-0086-x.

Havaei, M., Davy, A., Warde-Farley, D., Biard, A., Courville, A., Bengio, Y., Pal, C., Jodoin, P.M. and Larochelle, H. (2016) 'Brain tumor segmentation with deep neural networks', Med. Image Anal., Vol. 35, pp.81-31.

Işın, A., Direkoğlu, C. and Şah, M. (2016) 'Review of MRI-based brain tumor image segmentation using deep learning methods' Procedia Computer Sci., August, Vol. 102, pp.317-324.

Islam, A., Reza, S.M.S. and Iftekharuddin, K.M. (2013) 'Multifractal texture estimation for detection and segmentation of brain tumors', IEEE Trans. on Biomedical Engg., November, Vol. 60, No. 11, pp.3204-3215.

Islam, J. and Zhang, Y. (2018) 'Brain MRI analysis for Alzheimer's disease diagnosis using an ensemble system of deep convolutional neural networks', Int. J. on Brain Informatics, Vol. 5, No. 2, doi:10.1186/s40708-018-0080-3.

Joseph, R.P., Singh, C.S. and Manikandan, M. (2014) 'Brain tumor MRI image segmentation and detection in image processing', Int. J. Res. Eng. Technol., Vol. 3, No. 1, pp.1-5.

Kalaiselvi, T., Nagaraja, P. and Sriramakrishnan, P. (2016) 'A simple image processing approach to abnormal slices detection from MRI tumor volumes', Int. J. Multimedia it's Appl., Vol. 8, No. 1, pp.55-64.

Khalil, M., Ayad, H. and Adib, A. (2018) 'Performance evaluation of feature extraction techniques in MR-brain image classification system', Int. Conference on Computational Modeling and Security, Vol. 127, pp.218-225.

Kharrat, A., Gasmi, K., Ben Messaoud, M., Benamrane, N. and Abid, M. (2010) 'A hybrid approach for automatic classification of brain MRI using genetic algorithm and support vector machine', Leonardo Journal of Sciences, July-December, Vol. 17, pp.71-82.

Kumar, P. and Vijayakumar, B. (2015) 'Brain tumour Mr image segmentation and classification using by PCA and RBF kernel based support vector machine', Middle-East Journal of Scientific Research, Vol. 23, No. 9, pp.2106-2116.

Laddha, R.R. and Ladhake, S.A. (2014) 'Brain tumor detection using morphological and watershed operators', International Journal of Application or Innovation in Engineering \& Management (IJAIEM), Vol. 3, No. 3, pp.383-387.

Lienhart, R. and Maydt, J. (2002) "'An extended set of Haar-like features for rapid object detection', ICIP02, pp.I.900-I.903.

Lindeberg, T. (2014) 'Scale selection', in Ikeuchi, K. (Ed.): Computer Vision: A Reference Guide, pp.701-713, Springer.

Logeswari, T. and Karnan, M. (2010) 'An improved implementation of brain tumor detection using segmentation based on soft computing', Journal of Cancer Research and Experimental Oncology, March, Vol. 2, No. 1, pp.6-14.

Lowe, D.G. (2004) 'Distinctive image features from scale-invariant keypoints', IJCV, Vol. 60, No. 2, pp.91-110.

Mallikarjuna, E. (2015) 'Brain tumor detection using segmentation based object labeling algorithm', J. Computer. Biosci. Eng., Vol. 3, No. 2, pp.1-3.

Matthew, A.R., Prasad, A. and Anto, P.B. (2017) 'A review on feature extraction techniques for tumor detection and classification from brain MRI', 2017 International Conference on Intelligent Computing, Instrumentation and Control Technologies (ICICICT). 
Meera, S. and Sindhu, A. (2015) 'A survey on detecting brain tumor in MRI images using image processing techniques', International Journal of Innovative Research in Computer and Communication Engineering, Vol. 3, No. 1, pp.123-129.

Menze, B., Jakab, A. and Bauer, S. (2014) 'The multimodal brain tumor image segmentation benchmark (BRATS)', IEEE Trans. Med. Imaging, October, Vol. 34, No. 10, pp.1-32.

Mohan, R.R., Ambily, P.K. and James, S.P. (2015) 'Brain tumor detection using image fusion and neural network', Int. J. Eng. Res. Gen. Sci., March-April, Vol. 3, No. 2.

Mustafa, I.D. and Hassan, M.A. (2016) 'A comparison between different segmentation techniques used in medical imaging', Am. J. Biomed. Eng., Vol. 6, No. 2, pp.59-69.

Mustaqeem, A. and Javed, A. (2012) 'An efficient brain tumor detection algorithm using watershed $\&$ thresholding based segmentation', I.J. Image, Graphics and Signal Processing, September, Vol. 10, pp.34-39.

Nabizadeh, N. and Kubat, M. (2015) 'Brain tumors detection and segmentation in MR images: Gabor wavelet vs. statistical features', Comput. Electr. Eng., March, Vol. 45, pp.286-301.

Nagarajan, G., Minu, R.I., Muthukumar, B., Vednarayanan, V. and Sundarsingh, S.D. (2016) 'Hybrid genetic algorithm for medical image feature extraction and selection', Int. Conference on Computational Modeling and Security, Vol. 85, pp.455-462.

Naik, J. and Patel, P.S. (2013) 'Tumor detection and classification using decision tree in brain MRI', International Journal of Computer Science and Network Security (IJCSNS), June, Vol. 14, No. 6, pp.49-53.

Nakhmani, A., Kikinis, R. and Tannenbaum, A. (2014) 'MRI brain tumor segmentation and necrosis detection using adaptive Sobolev snakes', in Proc. SPIE Int. Soc. Opt. Eng., pp.1-10.

Neuroimaging Tools \& Resources Collaboratory [online] https://www.nitrc.org/ (accessed 05/01/2019).

Nisar, S., Khan, M.M., Ibrahim, M. and Tariq, M. (2015) 'Efficient detection of brain tumor using normalized histogram', J. Basic Appl. Sci. Res., Vol. 5, No. 4, pp.34-43.

Padmavathi, K. and Megala, M.C. (2015) 'Detection of brain tumor with filtering techniques', International J. Adv. Res. Computer Commune. Eng., Vol. 4, No. 7, pp.590-593.

ParthaSarathi, M. and Ansari, M.A. (2017) 'Multimodal retrieval framework for brain volumes in 3D MR volumes', J. Med. Biol. Eng., DOI: 10.1007/s40846-017-0287-4.

Patil, M.D., Chaithra, S., Divya, Dixit, S. and Kini, M.R. (2016) 'Brain tumor detection from clinical CT and MRI using wavelet based image fusion technique', Indian J. Res., Vol. 5, No. 6, pp.284-286.

Poonam, J.P. (2014) 'Review of image processing techniques for automatic detection of tumor in human brain', Int. J. Comput. Sci. Mob. Comput., Vol. 3, No. 3, pp.371-378.

Rajesh, D.A.S.B. and Patil, C. (2013) 'Brain tumour extraction from MRI images using MATLAB', Int. J. Electron. Commun. Soft Comput. Sci. Eng., Vol. 2, No. 1, pp.2-5.

Sánchez, J., Monzón, N. and Salgado, A. (2018) 'An Analysis and Implementation of the Harris corner detector', Image Processing on Line (IPOL), Vol. 8, pp.305-328, DOI: 10.5201/ipol. 2018.229.

Selkar, R.G. and Thakare, P. (2014) 'Brain tumor detection and segmentation by using thresholding and watershed algorithm', International Journal of Advance Information and Communication Technology, Vol. 1, No. 3, pp.321-324.

Shree, N.V. and Kumar, T.N.R. (2018) 'Identification and classification of brain tumor MRI images with feature extraction using DWT and probabilistic neural network', Int. J. on Brain Informatics, March, Vol. 5, No. 1, pp.23-30.

Silva, C., Bouwmans, T. and Frelicot, C. (2015) 'An extended center-symmetric local binary pattern for background modeling and subtraction in videos', VISAPP 2015, Berlin, Germany, March. 
Singh, G. and Ansari, M.A. (2016) 'Efficient detection of brain tumor from MRIs using K-means $\backslash$ segmentation and normalized histogram', IEEE 1st India International Conference on Information Processing (IICIP).

Subashini, M.M. and Sahoo, S.K. (2013) 'Brain MR image segmentation for tumor detection using artificial neural networks', Int. J. Eng. Technol., Vol. 5, No. 2, pp.925-933.

Subashini, M.M., Sahoo, S.K., Sunil, V. and Easwaran, S. (2016) 'A non-invasive methodology for the grade identification of astrocytoma using image processing and artificial intelligence techniques', Expert System with Applications, Vol. 43, pp.186-196, Elsevier.

Subramanyam, M. and Raviraj, P. (2015) 'An automatic brain tumor detection and segmentation scheme for clinical brain images', Int. J. Emerg. Technol. Comput. Appl. Sci., March-May, Vol. 8, No. 1, pp.37-42.

Vidyarthi, A. and Mittal, N. (2015) 'Performance analysis of Gabor-wavelet based features in classification of high grade malignant brain tumors', IEEE 39th National System Conference, December.

Yang, A.M., Yang, X.L., Wu, W.R., Liu, H.X. and Zhuansun, Y.X. (2019) 'Research on feature extraction of tumor image based on convolutional neural network', IEEE Access, Vol. 7.

Zanaty, E.A. (2012) 'Determination of gray matter (GM) and white matter (WM) volume in brain magnetic resonance images (MRI)', International Journal of Computer Applications, Vol. 45, No. 3, pp.16-22.

Zulpe, N. and Pawar, V. (2012) 'GLCM textural features for brain tumor classification', International Journal of Computer Science Issues, May, Vol. 9, No. 3, pp.354-359. 